ARTigo ESPECIAL

Special Article

\title{
Lúpus Eritematoso Sistêmico: Tratamento do Acometimento Sistêmico(*)
}

\author{
Sato EI, Bonfá ED, Costallat LTL, Silva NA, Brenol JCT, Santiago MB, Szajubok JCM, \\ Rachid-Filho A, Barros RT, Vasconcelos M
}

\section{DESCRIÇÃO DO MÉTODO DE COLETA DE EVIDÊNCIAS}

Oito reumatologistas que trabalham em serviços que atendem grande número de pacientes com lúpus eritematoso sistêmico, alguns dos quais têm pesquisa e publicações científicas nesta área, foram convidados a participar do grupo de trabalho. Também foram convidados um nefrologista e uma dermatologista que atuam em grandes centros universitários, com grande experiência no atendimento destes pacientes. Todos se reuniram para discutir o tratamento das diferentes manifestações da doença, subdivididos em quatro grupos de trabalho, cada qual ficando responsável por buscar a melhor evidência para o tratamento de um ou mais comprometimentos da doença. A última edição de Dubois's lupus erythematosus, editado por Wallace D e Hahn B, em 2001, foi utilizada como base da discussão. Trabalhos publicados nos últimos cinco anos foram pesquisados no banco de dados do Medline. Devido à freqüência e à heterogeneidade de manifestações da doença, a maioria dos trabalhos terapêuticos não contempla grande casuística, e nem são randômicos e controlados. Como as manifestações e a gravidade da doença variam em diferentes grupos populacionais, devemos avaliar com cuidado os estudos realizados em grupos populacionais distintos.

\section{GRAU DE RECOMENDAÇÃO E FORÇA DE EVIDÊNCIA}

A: Estudos experimentais e observacionais de melhor consistência.

B: Estudos experimentais e observacionais de menor consistência.

C: Relatos de casos (estudos não controlados).

D: Opinião desprovida de avaliação crítica, baseada em consensos, estudos fisiológicos ou modelos animais.

\section{OBJETIVOS}

Elaborar recomendação baseada na melhor evidência científica para o tratamento das diversas manifestações sistêmicas do lúpus eritematoso sistêmico.

\section{CONFLITO DE INTERESSE}

Nenhum conflito de interesse declarado.

\section{COMPROMETIMENTO HEMATOLÓGICO}

Não há estudos controlados avaliando o tratamento das anemias hemolíticas auto-imunes. O tratamento de escolha é feito com prednisona em dose alta, ou muito alta, por quatro a seis semanas com posterior redução, na dependência da resposta. Setenta e cinco por cento dos casos respondem satisfatoriamente a esta terapêutica ${ }^{(1)}($ D) . A pulsoterapia com metilprednisolona deve ser indicada para casos graves, em que se requer uma resposta mais rápida e a manutenção é feita com prednisona por via oral em dose baixa $^{(2)}(\mathrm{D})$.

Nos casos refratários à corticoterapia, ou quando da necessidade de altas doses de manutenção, pode-se associar 1-3 $\mathrm{mg} / \mathrm{kg} /$ dia de azatioprina à prednisona em baixas doses $^{(3)}(\mathbf{D})$ ou danazol associado à prednisona ${ }^{(4)}(\mathbf{B})$. Imunoglobulina intravenosa mostrou ser eficaz em pacientes com anemia hemolítica auto-imune, podendo ser indicada em casos muito específicos como os que têm contra-indicação ou toxicidade com outras terapias ${ }^{(5)}(\mathbf{C})$.

* Trabalho realizado por representantes da Sociedade Brasileira de Reumatologia. Elaboração final: 8 de junho de 2004.

O Projeto Diretrizes, iniciativa conjunta da Associação Médica Brasileira e Conselho Federal de Medicina, tem por objetivo conciliar informações da área médica a fim de padronizar condutas que auxiliem o raciocínio e a tomada de decisão do médico. As informações contidas neste projeto devem ser submetidas à avaliação e à crítica do médico, responsável pela conduta a ser seguida, frente à realidade e ao estado clínico de cada paciente a ser seguida, frente à realidade e ao estado clínico de cada paciente. 
As plaquetopenias leves, contagem menor que 50.000, geralmente não requerem tratamento específico ${ }^{(1)}(\mathrm{D})$. Prednisona em dose alta é o tratamento de escolha para plaquetopenia sintomática. Pulsoterapia com metilprednisolona pode ser utilizada para plaquetopenia grave, quando se requer resposta mais rápida, embora sua superioridade em relação ao uso de glicocorticóides oral não tenha sido confirmada $^{(1)}($ D). Na falta de resposta aos glicocorticóides, danazol, um esteróide androgênico com poucos efeitos virilizantes utilizado na dose de manutenção de 200 a 400 $\mathrm{mg} /$ dia, após dose inicial mais elevada, se mostrou efetivo $^{(6)}(\mathbf{A})$. Outras drogas que têm mostrado alguma eficácia em pequeno número de casos com trombocitopenia refratária são: ciclofosfamida ${ }^{(7)}(\mathbf{C})$, antimaláricos associados à prednisona ${ }^{(8)}(\mathbf{C})$ e $1-3 \mathrm{mg} / \mathrm{kg} /$ dia de azatioprina ${ }^{(9)}(\mathbf{C})$.

Imunoglobulina intravenosa $-400 \mathrm{mg} / \mathrm{kg} / \mathrm{dia}$ - pode ser indicada ${ }^{(10)}(\mathbf{C})$ associada à infusão de plaquetas ${ }^{(11)}(\mathbf{C})$ nas plaquetopenias graves, com risco de vida. Seu custo extremamente alto deve ser considerado.

A efetividade da esplenectomia em trombocitopenia refratária a glicocorticóides é controversa. Um relato de caso mostrou resposta favorável e persistente ${ }^{(8)}(\mathbf{C})$, no entanto, a esplenectomia só deve ser indicada depois da tentativa de outras terapias medicamentosas ${ }^{(1)}(\mathbf{D})$.

\section{COMPROMETIMENTO CARDIOPULMONAR}

O envolvimento das túnicas cardíacas geralmente apresenta boa resposta à corticoterapia, em doses moderadas, com exceção das miocardiopatias, que em alguns casos podem ter resposta inadequada ${ }^{(12)}(\mathbf{D})$. Nos raros casos de tamponamento cardíaco, é indicada a pericardiocentese. Há evidências fisiopatológicas de risco de aterosclerose em virtude do aumento da sobrevida, do uso crônico de corticosteróides, além do papel da inflamação na gênese da placa aterosclerótica. O paciente deve ser orientado a controlar fatores de risco associados à doença coronária como dieta, tabagismo, obesidade, sedentarismo e níveis lipídicos sangüíneos. Os antimaláricos têm efeito benéfico no controle da dislipidemia desses pacientes ${ }^{(13)}(\mathbf{B})$.

A pleurite geralmente é responsiva a corticosteróides em doses moderadas. $\mathrm{O}$ comprometimento parenquimatoso, embora mais raro, é muito grave, principalmente a pneumonite aguda e a hemorragia pulmonar ${ }^{(14)}(\mathbf{C})$. Nestes casos está indicada a imunossupressão vigorosa com corticosteróides $^{(15)}(\mathbf{C})$. O tratamento da hipertensão pulmonar também requer o uso de imunossupressores, a ciclofosfamida é efetiva quando iniciada precocemente ${ }^{(16)}(\mathbf{C})$. Resultados modestos podem ser obtidos com anticoagulantes, prostaciclinas e agentes vasodilatadores, como a nifedipina.

\section{MANIFESTAÇÕES NEUROPSIQUIÁTRICAS}

O diagnóstico envolve a exclusão rigorosa de eventos secundários a outras situações clínicas que podem ocorrer, como distúrbios metabólicos, infecções ou uso de medicamentos. $\mathrm{O}$ espectro das manifestações neuropsiquiátricas é muito variável, podendo acometer os sistemas nervosos central e periférico. Em 1999, o subcomitê do Colégio Americano de Reumatologia classificou 19 síndromes neuropsiquiátricas relacionadas à doença lúpica ${ }^{(17)}(D)$. (Tabela 1).

O tratamento das manifestações neuropsiquiátricas deverá ser dirigido ao tipo de manifestação apresentada. Embora não haja estudos terapêuticos controlados, podem ser tratadas com glicocorticóides e/ou imunossupressores, preferencialmente a ciclofosfamida ${ }^{(18)}(\mathbf{B})$, em doses que variam de acordo com a gravidade.

Nos casos de doenças cerebrovasculares, em decorrência de fenômenos tromboembólicos, muitas vezes relacionados aos anticorpos antifosfolípides, o uso de anticoagulantes está indicado $^{(18)}($ D $)$. A tomografia computadorizada cerebral e, principalmente, a ressonância nuclear magnética com gadolínio auxiliam na detecção do envolvimento do sistema nervoso central e da medula espinhal. Estudos comparando os dois métodos em pacientes com manifestações neuropsiquiátricas têm comprovado a superioridade da ressonân$\mathrm{cia}^{(20)}(\mathbf{C})$. Para o tratamento das convulsões podem ser prescritos anticonvulsivantes, assim como na psicose os antipsicóticos podem ser bons coadjuvantes ${ }^{(19)}(\mathbf{D})$.

A psicose é, de modo geral, fruto do dano imunológico da doença, embora possa, mais raramente, ser secundária à corticoterapia. O paciente também pode apresentar desordens do humor, de ansiedade e distúrbios cognitivos, como déficit de memória e de atenção, dificuldade no aprendizado e no raciocínio, dentre outros que devem ser adequadamente avaliados e tratados pelo especialista.

Mononeuropatia e polineuropatia periférica geralmente respondem ao esquema de corticosteróides e/ou imunossupressores $^{(19)}(\mathbf{D})$. Neuropatia craniana ocorre, em geral, em doença ativa, é transitória e responde ao tratamento convencional com glicocorticóides em altas doses ${ }^{(19)}(\mathbf{D})$. A mielopatia, que pode ser transversa e cujo diagnóstico é melhor realizado pela ressonância, deve ser tratada com pulsoterapia de corticosteróides associada à ciclofosfamida endovenosa $1 \mathrm{~g} / \mathrm{m}^{2}$ superfície corpórea ${ }^{(21)}(\mathbf{B})$. 
TABELA 1

SÍNDROMES NEUROPSIQUIÁTRICAS RELACIONADAS AO LÚPUS ERITEMATOSO SISTÊMICO ${ }^{(17)}($ D $)$

\begin{tabular}{l} 
Sistema nervoso central \\
- Estado confusional agudo \\
- Distúrbios cognitivos \\
- Psicose \\
- Desordens de humor \\
- Desordens de ansiedade \\
- Cefaléia \\
- Doença cerebrovascular \\
- Mielopatia \\
- Desordens do movimento \\
- Síndromes desmielinizantes \\
- Convulsões \\
- Meningite asséptica \\
Sistema nervoso periférico \\
- Neuropatia craniana \\
- Polineuropatia \\
- Plexopatia \\
- Mononeuropatia simples/múltipla \\
- Polirradiculoneuropatia inflamatória aguda (Guillain-Barré) \\
- Desordens autonômicas \\
\hline
\end{tabular}

A cefaléia é um sintoma muito freqüente e pode ser decorrente de outras causas, independente do lúpus eritematoso sistêmico, e, portanto, a terapêutica específica nestes casos deve ser bem avaliada.

Nos casos com comprometimento neurológico grave, não responsivos à corticoterapia, uso de ciclofosfamida e/ ou da anticoagulação nos casos de trombose, pode-se indicar plasmaferese ou imunoglobulina endovenosa, mas, na literatura, os resultados são controversos ${ }^{(19)}(D)$. Outros imunossupressores como a azatioprina e metotrexato não têm mostrado efeito similar ao da ciclofosfamida nestes $\operatorname{casos}^{(21)}(\mathbf{B})$.

\section{COMPROMETIMENTO RENAL}

Os principais objetivos do tratamento da nefropatia são: controlar a atividade inflamatória e prevenir a evolução para a insuficiência renal crônica. Os parâmetros utilizados para avaliar o envolvimento renal e o monitoramento da terapêutica são $^{(22)}(\mathbf{D})$ :
Clínico: edema, oligúria e hipertensão arterial, em número significativo de casos pode estar assintomática;

Laboratorial: exame do sedimento urinário, proteinúria de 24 horas, creatinina e albumina séricas, depuração de creatinina, C3 e anti-DNAds;

Biópsia Renal: É desejável sua realização em pacientes com envolvimento renal, caracterizado por: sedimento urinário anormal (hematúria e cilindrúria) e/ou proteinúria maior que $1 \mathrm{~g} / 24 \mathrm{~h}$ e/ou alteração da função renal, excluindo-se sua indicação na nefropatia crônica terminal. Este procedimento deverá ser realizado em centros de referência, com profissionais experientes e habilitados ${ }^{(23)}(\mathbf{D})$.

\section{MEDIDAS GERAIS NA NEFROPATIA}

1. Controle rigoroso da hipertensão arterial, estando também indicados os agentes inibidores da angiotensina, tendo em vista seus efeitos antiproteinúricos e renoprotetores, exceto quando ocorrer disfunção renal aguda ${ }^{(24)}(\mathbf{A})$;

2. Outras: controle da obesidade e da dislipidemia, interrupção do tabagismo, restrição ao uso de antiinflamatórios não esteróides e outras drogas potencialmente nefrotó$\operatorname{xicas}^{(25)}(\mathrm{D})$.

\section{MEDIDAS MEDICAMENTOSAS}

1. Para os pacientes com glomerulonefrite proliferativa, classes III e IV da OMS, o glicocorticóide é a droga de escolha para a doença ativa, na dose de 1 a $1,5 \mathrm{mg} / \mathrm{kg} / \mathrm{dia}$ de prednisona, por um período de seis a oito semanas, seguindo-se sua redução progressiva até $0,25 \mathrm{mg} / \mathrm{kg}$ / $\operatorname{dia}^{(26)}(\mathbf{C})$. A pulsoterapia com metilprednisolona está indicada para os casos mais graves, com disfunção renal aguda. A dose recomendada é de 500 a $1.000 \mathrm{mg} /$ dia por três dias consecutivos, por via endovenosa ${ }^{(27)}(\mathbf{B})$;

2. Nas nefrites proliferativas está indicada a associação de glicocorticóides com agentes imunossupressores citostáticos, particularmente a ciclofosfamida por via oral ou endovenosa, sob a forma de pulsos inicialmente mensais, e posteriormente bimestrais ou trimestrais ${ }^{(28)}(\mathbf{A})$. Alternativamente, drogas eficazes e menos tóxicas poderão ser indicadas nos casos sem disfunção renal importante tais como: micofenolato de mofetil ${ }^{(29)}(\mathbf{A})$, esquema seqüencial de dose baixa de ciclofosfamida e azatioprina ${ }^{(30)}(\mathbf{A})$ e imunoglobulina endovenosa ${ }^{(31)}($ D) . A despeito de algumas controvérsias, até o momento, a ciclofosfamida é considerada a terapia mais efetiva para o tratamento inicial de nefrite lúpica grave, entretanto, como esta não é capaz de controlar todos os casos e em razão de sua toxicidade, novos esquemas terapêuticos devem ser avaliados ${ }^{(32)}(\mathbf{D})$. Estudos de meta-análise 
evidenciaram a superioridade do uso associado de imunossupressores na preservação da função renal e na sobrevida dos pacientes com nefrite lúpica, quando comparado ao uso isolado de glicocorticosteróides ${ }^{(33)}(\mathbf{A})$, sem que se comprovasse superioridade de um imunossupressor sobre outro $^{(34)}(\mathbf{A})$. Mais recentemente, na tentativa de diminuir os efeitos colaterais decorrentes do uso prolongado de ciclofosfamida, um estudo mostrou melhores resultados com uso de azatioprina ou de micofenolato mofetil como drogas de manutenção, após a indução da remissão da nefrite lúpica proliferativa com o esquema clássico de ciclofosfamida intravenosa por seis meses ${ }^{(35)}(\mathbf{A})$;

3. Nos pacientes com nefrite membranosa, classe $\mathrm{V}$ da OMS, o tratamento é controverso. Podem ser utilizados glicocorticóides e/ou agentes imunossupressores, na dependência do quadro de síndrome nefrótica ${ }^{(36)}(\mathbf{D})$. Entre os imunossupressores podem ser indicados: ciclofosfamida $\operatorname{oral}^{(37)}(\mathbf{B})$, ciclosporina $^{(38)}(\mathbf{C})$ e azatioprina ${ }^{(39)}(\mathbf{B})$;

4. Na situação de evolução para a perda cronicamente evolutiva da função renal, com depuração de creatinina menor que $30 \mathrm{ml} / \mathrm{min}$, não estará indicada a terapia imunossupressora, com glicocorticóides ou citostáticos, exceto para o tratamento de manifestações extra-renais;

5. A terapêutica substitutiva renal poderá incluir a utilização de métodos dialíticos e o transplante renal. A indicação deste último procedimento deverá obedecer a prazo não inferior a um ano de remissão da atividade da doença ${ }^{(40)}($ D) .

\section{GRAVIDEZ , ANTICONCEPÇÃO E REPOSIÇÃO HORMONAL}

\section{FERTILIDADE E CONCEPÇÃO}

A fertilidade no lúpus é normal e a gravidez, embora não contra-indicada, deve ser planejada. Considerar atividade e gravidade da doença e toxicidade das drogas. A gravidez é contra-indicada em casos de nefropatia lúpica em atividade $^{(41)}(\mathbf{C})$.

\section{MONITORAMENTO}

A gravidez nas pacientes com lúpus eritematoso sistêmico deve ser considerada de alto risco, necessitando acompanhamento multidisciplinar, até o puerpério, devido à possibilidade de reagudização. $\mathrm{Na}$ gravidez, podem ocorrer eritemas palmar e facial, artralgias e elevação da VHS, dificultando a caracterização da atividade da doença. A presença do anti-DNAds e/ou elevação dos seus títulos e con- sumo do complemento podem auxiliar na diferenciação entre atividade de doença e pré-eclâmpsia ${ }^{(42)}(\mathbf{D})$.

É importante a pesquisa dos anticorpos antifosfolípides, anti-Ro/SS-A e anti-La/SS-B devido à possibilidade da ocorrência da síndrome antifosfolípide ${ }^{(43)}(\boldsymbol{D})$ e do lúpus neonatal ${ }^{(4)}(D)$, respectivamente.

\section{COMPLICAÇÕES}

Existe uma maior incidência de retardo de crescimento intra-uterino, prematuridade, hipertensão induzida pela gravidez e diabetes $^{(45)}(\mathbf{D})$.

Deve-se estar atento à possibilidade de insuficiência supra-renal na mãe e no feto no pós-parto imediato.

\section{ALEITAMENTO}

Doses de glicocorticóides superiores a $20 \mathrm{mg} /$ dia de prednisona podem determinar riscos para a criança, sendo recomendado um intervalo de 4 horas entre a tomada da medicação e a amamentação ${ }^{(46)}(\mathbf{C})$. Quando inevitável o uso de imunossupressores, a opção é a azatioprina em baixas doses $^{(47)}($ D). O controle da atividade da doença deve ser feito com glicocorticóides (prednisona). Outras drogas que podem ser utilizadas nos casos refratários são: azatioprina, micofenolato de mofetil, ciclosporina e imunoglobulina endovenosa $^{(48)}($ D) .

$\mathrm{Na}$ síndrome antifosfolípide com história de perda fetal, está indicado o uso de heparina e aspirina. Recomenda-se o uso de aspirina em baixas doses na presença do anticorpo antifosfolípide, em títulos moderados ou elevados ${ }^{(49)}(\mathbf{D})$.

\section{ANTICONCEPÇÃO E TERAPIA DE REPOSIÇÃO HORMONAL}

A anticoncepção pode ser feita com progesterona e métodos de barreira ou com associação de ambos.

A utilização de estrógenos em baixas doses na anticoncepção e na reposição permanece controversa ${ }^{(50)}(\mathrm{D})$. Na síndrome antifosfolípide, seu uso é contra-indicado ${ }^{(51)}(\mathrm{D})$.

\section{VACINAÇÃO}

As vacinas contra o pneumococo ${ }^{(52)}(\mathbf{A})$ e influenza ${ }^{(53)}(\mathbf{B})$ são seguras e têm uma eficácia quase semelhante à da população normal. Entretanto, não devem ser administradas nos períodos de atividade da doença. As vacinas com vírus vivos não devem ser prescritas a pacientes com lúpus eritematoso sistêmico. Não estão determinadas a segurança e a eficácia da vacina contra hepatite $\mathrm{B}$ nestes pacientes. 


\section{REFERÊNCIAS}

1. Quismorio FP Jr. Hematologic and lymphoid abnormalities in systemic lupus erythematosus. In: Wallace DJ, Hahn BH, editors. Dubois lupus erythematosus. Philadelphia: Lippincott Williams \& Wilkins; 2002. p.793-819.

2. Jacob HS. Pulse steroids in hematologic diseases. Hosp Pract (Off Ed) 20:87-94, 1985.

3. Pirofsky B. Immune haemolytic disease: the autoimmune haemolytic anaemias. Clin Haematol 4:167-80, 1975.

4. Pignon JM, Poirson E, Rochant H. Danazol in autoimmune haemolytic anaemia. Br J Haematol 83:343-5, 1993.

5. Majer RV, Hyde RD. High-dose intravenous immunoglobulin in the treatment of autoimmune haemolytic anaemia. Clin Lab Haematol 10:391-5, 1988.

6. Cervera H, Jara LJ, Pizarro S, et al. Danazol for systemic lupus erythematosus with refractory autoimmune thrombocytopenia or Evans syndrome. J Rheumatol 22:1867-71, 1995.

7. Roach BA, Hutchinson GJ. Treatment of refractory, systemic lupus erythematosus-associated thrombocytopenia with intermittent lowdose intravenous cyclophosphamide. Arthritis Rheum 36:682-4, 1993.

8. Arnal C, Piette JC, Leone J, et al. Treatment of severe immune thrombocytopenia associated with systemic lupus erythematosus: 59 cases. J Rheumatol 29:75-83, 2002.

9. Goebel KM, Gassel WD, Goebel FD. Evaluation of azathioprine in autoimmune thrombocytopenia and lupus erythematosus. Scand J Haematol 10:28-34, 1973.

10. Ter Borg EJ, Kallenberg CG. Treatment of severe thrombocytopenia in systemic lupus erythematosus with intravenous gammaglobulin. Ann Rheum Dis 51:1149-51, 1992.

11. Chandramouli NB, Rodgers GM. Prolonged immunoglobulin and platelet infusion for treatment of immune thrombocytopenia. Am J Hematol 65:85-6, 2000.

12. D'Cruz D, Khamashta M, Hughes G. Cardiovascular manifestations of systemic lupus erythematosus. In: Wallace DJ, Hahn BH, editors. Dubois lupus erythematosus. Philadelphia: Lippincott Williams \& Wilkins; 2002. p.645-61.

13. Borba EF, Bonfa E. Long term beneficial effect of chloroquine diphosphate on lipoprotein profile in lupus patients with and without steroid therapy. J Rheumatol 28:780-5, 2001.

14. Carette S, Macher AM, Nussbaum A, Plotz PH. Severe, acute pulmonary disease in patients with systemic lupus erythematosus: ten years of experience at the National Institutes of Health. Semin Arthritis Rheum 14:52-9, 1984.

15. Zamora MR, Warner ML, Tuder R, Schwarz MI. Diffuse alveolar hemorrhage and systemic lupus erythematosus: clinical presentation, histology, survival, and outcome. Medicine (Baltimore) 76:192-202, 1997.

16. Tanaka E, Harigai M, Tanaka M, Kawaguchi $Y$, Hara M, Kamatani N. Pulmonary hypertension in systemic lupus erythematosus: evaluation of clinical characteristics and response to immunosuppressive treatment. J Rheumatol 29:282-7, 2002.

17. The American College of Rheumatology nomenclature and case definitions for neuropsychiatric lupus syndromes. Arthritis Rheum 42:599-608, 1999.

18. Boumpas DT, Yamada H, Patronas NJ, Scott D, Klippel JH, Balow JE. Pulse cyclophosphamide for severe neuropsychiatric lupus. Q J Med 81:975-84, 1991.
19. West SG. Systemic lupus erythematosus and the nervous system. In: Wallace DJ, Hahn BH, editors. Dubois lupus erythematosus. Philadelphia: Lippincott Williams \& Wilkins; 2002. p.693-738.

20. Sibbitt WL Jr, Sibbitt RR, Griffey RH, Eckel C, Bankhurst AD. Magnetic resonance and computed tomographic imaging in the evaluation of acute neuropsychiatric disease in systemic lupus erythematosus. Ann Rheum Dis 48:1014-22, 1989.

21. Barile $\mathrm{L}$, Lavalle C. Transverse myelitis in systemic lupus erythematosus - the effect of IV pulse methylprednisolone and cyclophosphamide. J Rheumatol 19:370-2, 1992.

22. Wallace DJ, Hahn BH, Klippel JH. Clinical and laboratory features of lupus nephritis. In: Wallace DJ, Hahn BH, editors. Dubois lupus erythematosus. Philadelphia: Lippincott Williams \& Wilkins; 2002. p. 1077-91.

23. Grande JP, Ballow JE. Renal biopsy in lupus nephritis. Lupus 7:611-7, 1998.

24. Ruggenenti P, Perna A, Gherardi G, Gaspari F, Benini R, Remuzzi G. Renal function and requirement for dialysis in chronic nephropathy patients on long-term ramipril: REIN follow-up trial. Gruppo Italiano di Studi Epidemiologici in Nefrologia (GISEN). Ramipril Efficacy in Nephropathy. Lancet 352:1252-6, 1998.

25. Praga M. Slowing the progression of renal failure. Kidney Int Suppl $80: 18-22,2002$.

26. Pollak VE. Treatment of lupus nephritis. Nippon Jinzo Gakkai Shi 25:776-85, 1983.

27. Kimberly RP, Lockshin MD, Sherman RL, McDougal JS, Inman RD, Christian CL. High-dose intravenous methylprednisolone pulse therapy in systemic lupus erythematosus. Am J Med 70:817-24, 1981.

28. Austin HA 3rd, Klippel JH, Balow JE, et al. Therapy of lupus nephritis: controlled trial of prednisone and cytotoxic drugs. N Engl J Med 314:614-9, 1986.

29. Chan TM, Li FK, Tang CS, et al. Efficacy of mycophenolate mofetil in patients with diffuse proliferative lupus nephritis. Hong KongGuangzhou Nephrology Study Group. N Engl J Med 343:1156-62, 2000 .

30. Houssiau FA, Vasconcelos C, D'Cruz D, et al. Immunosuppressive therapy in lupus nephritis: the Euro-Lupus Nephritis Trial, a randomized trial of low-dose versus high-dose intravenous cyclophosphamide. Arthritis Rheum 46:2121-31, 2002.

31. Rauova L, Lukac J, Levy Y, Rovensky J, Shoenfeld Y. High-dose intravenous immunoglobulins for lupus nephritis: a salvage immunomodulation. Lupus 10:209-13, 2001.

32. Balow JE. Choosing treatment for proliferative lupus nephritis. Arthritis Rheum 46:1981-3, 2002.

33. Flanc RS, Roberts MA, Strippoli GF, Chadban SJ, Kerr PG, Atkins RC. Treatment of diffuse proliferative lupus nephritis: a meta-analysis of randomized controlled trials. Am J Kidney Dis 43:197-208, 2004.

34. Bansal VK, Beto JA. Treatment of lupus nephritis: a meta-analysis of clinical trials. Am J Kidney Dis 29:193-9, 1997.

35. Contreras G, Pardo V, Leclercq B, et al. Sequential therapies for proliferative lupus nephritis. N Engl J Med 350:971-80, 2004.

36. Kolasinski SL, Chung JB, Albert DA. What do we know about lupus membranous nephropathy? An analytic review. Arthritis Rheum 47:450-5, 2002.

37. Chan TM, Li FK, Hao WK, et al. Treatment of membranous lupus nephritis with nephrotic syndrome by sequential immunosuppression. Lupus 8:545-51, 1999. 
38. Radhakrishnan J, Kunis CL, D'Agati V, Appel GB. Cyclosporine treatment of lupus membranous nephropathy. Clin Nephrol 42: 147-54, 1994.

39. Mok CC, Ying KY, Lau CS, et al. Treatment of pure membranous lupus nephropathy with prednisone and azathioprine: an open-label trial. Am J Kidney Dis 43:269-76, 2004.

40. Stone JH. End-stage renal disease in lupus: disease activity, dialysis, and the outcome of transplantation. Lupus 7:654-9, 1998.

41. Urowitz MB, Gladman DD, Farewell VT, Stewart J, McDonald J. Lupus and pregnancy studies. Arthritis Rheum 36:1392-7,1993.

42. Buyon JP, Kalunian KC, Ramsey-Goldman R, Petri MA, Lockshin $\mathrm{MD}$, Ruiz-Irastorza G, et al. Assessing disease activity in SLE patients during pregnancy. Lupus 8:677-84, 1999.

43. Wilson WA, Gharavi AE, Koike $\mathrm{T}$, et al. International consensus statement on preliminary classification criteria for definite antiphospholipid syndrome: report of an international workshop. Arthritis Rheum 42:1309-11, 1999.

44. Buyon JP, Clancy RM. Neonatal lupus syndromes. Curr Opin Rheumatol 15:535-41, 2003.

45. Cervera R, Font J, Carmona F, Balasch J. Pregnancy outcome in systemic lupus erythematosus: good news for the new millennium. Autoimmun Rev 1: 354-9, 2002.
46. Ost L, Wettrell G, Bjorkhem I, Rane A. Prednisolone excretion in human milk. J Pediatr 106:1008-11, 1985.

47. Anderson PO. Drugs and breast feeding. Semin Perinatol 3:271-8, 1979.

48. Bermas BL, Hill JA. Effects of immunosuppressive drugs during pregnancy. Arthritis Rheum 38:1722-32, 1995.

49. Ruiz-Irastorza G, Khamashta MA, Hughes GR. Antiaggregant and anticoagulant therapy in systemic lupus erythematosus and Hughes' syndrome. Lupus 10:241-5, 2001.

50. Petri M. Exogenous estrogen in systemic lupus erythematosus: oral contraceptives and hormone replacement therapy. Lupus 10:222-6, 2001.

51. Lahita RG. Hormonal contraception and replacement and the use of androgens in the antiphospholipid syndrome. J Autoimmun 15:213-6, 2000 .

52. Klippel JH, Karsh J, Stahl NI, Decker JL, Steinberg AD, Schiffman G. A controlled study of pneumococcal polysaccharide vaccine in systemic lupus erythematosus. Arthritis Rheum 22:1321-5, 1979.

53. Abu-Shakra M, Zalmanson S, Neumann L, Flusser D, Sukenik S, Buskila D. Influenza virus vaccination of patients with systemic lupus erythematosus: effects on disease activity. J Rheumatol 27:1681-5, 2000. 